\title{
Abortion and shedding of Brucella abortus RB51 in vaginal exudates of vaccinated cows
}

\author{
H. I. Hosein', M. N. Shalaby' ${ }^{2}$ R. A. Azzam ${ }^{1}$ \\ ${ }^{1}$ Deptartment of Veterinary Medicine, Faculty of Veterinary Medicine, Beni-Suef, University, Beni-Suef, Egypt \\ ${ }^{2}$ Animal Health Research Institute, Dokki, Giza, Egypt
}

\begin{abstract}
In this study, Brucella abortus RB51 could be detected in the vaginal exudates of 5 out of 26 aborted cows following vaccination of three dairy cattle herds of 3810 animals including heifers and pregnant and non-pregnant cows by RB51 vaccine. Abortion associated with isolation of RB51 vaccinal strain from recently vaccinated cows in this study served as evidence that the vaccinal RB51 strain was the cause of abortion due to extensive bacterial multiplication and colonization in the uterus of vaccinated pregnant cows.
\end{abstract}

In spite of the exhaustive control efforts implemented in the last twenty-five years in Egypt, brucellosis persists as a major threat to livestock industry and public health and remains as an enzootic disease.

The disease causes significant decrease in milk production, abortion and infertility that interfere with genetic improvement programs, (Blasco, 1998). In addition, the disease is readily transmissible to humans through direct and aerosol contact, (Covert et al., 2005) leading to reproductive, respiratory, cardio-vascular, lymphoid and CNS pathology.

The growing prevalence of brucella infection among livestock in Egypt is mainly attributed to the failure of control programs and consequently the continuous spread of the disease. In addition, introduction of infection from other countries due to importation of living animals which is allowed nowadays may occur. Moreover, as reservoir, stray $\operatorname{dogs}$ as well as rats may pose a great risk to livestock (Hosein et al., 2001).

In the developing countries, control of brucellosis is based on immunization of livestock and slaughtering of positive cases. However, vaccines are not $100 \%$ efficacious against all strains of brucella (Schurig et al., 2002)

Brucella abortus RB51 is a stable rough attenuated mutant derived from the virulent strain 2308 and is used as a vaccine. It does not express significant amount of the $\mathrm{O}$ polysaccharide chain (O-chain) of the lipopolysaccharide (Schurig et al., 1991).
Although some studies recommended the use of RB51 to protect cattle against brucellosis, most of them were done under low prevalence condition of brucellosis. Therefore, the efficacy of this vaccine remains controversial.

RB51 is the official brucella vaccine used in Egypt under the supervision of the veterinary authorities. The vaccine has been evaluated in many studies, which stated the failure of the vaccine to induce good protection against the virulent Brucella melitensis infection (Hosein et al., 2002 and El-Diasty, 2004).

The present work was carried out to investigate the cause of abortion in dairy cattle herds following RB51 vaccination.

\section{Material and methods}

Animals. A total of 26 aborted cows following RB51 vaccination out of a total of 3810 vaccinated cows were subjected to serological and bacteriological examination.

Samples. Blood serum samples, vaginal exudates and milk were collected from the 26 aborted cows.

Antigens. a. Rough antigen of RB51 was prepared according to (Mehanna, 1989). b. Antigens for TAT, BAPA test and Rivanol test were obtained from the Veterinary Serum and Vaccine Research Institute, Abbassia, Cairo, Egypt.

TAT, BAPAT and Rivanol test. were carried out according to (Alton et al., 1988).

RB51 vaccine. was obtained from the Professional Biological Company, license No. 188, Denver, USA. 
Table (1): Rate of abortion following RB51 vaccination.

\begin{tabular}{ccccc}
\hline Herds & $\begin{array}{c}\text { No. of vaccinated } \\
\text { animals }\end{array}$ & $\begin{array}{c}\text { Cases of } \\
\text { abortion }\end{array}$ & $\begin{array}{c}\text { Agglutination } \\
\text { using smooth } \\
\text { antigen }\end{array}$ & $\begin{array}{c}\text { Mean titers against rough } \\
\text { antigens }\end{array}$ \\
\hline Herd 1 & 960 & $\begin{array}{c}6 \\
(0.62 \%) \\
12\end{array}$ & - ve & $1 / 640$ \\
Herd 2 & 1200 & $(1.25 \%)$ & - ve & $1 / 640$ \\
& & 8 & & $1 / 320$ \\
Herd 3 & 1650 & $(0.83 \%)$ & $-\mathrm{ve}$ & - \\
Total & 3810 & $(0.68 \%)$ & $-\mathrm{ve}$ & \\
\hline
\end{tabular}

Table (2): Isolation of Brucella organisms from vaginal exudates of aborted cows.

\begin{tabular}{lccc}
\hline \multirow{2}{*}{ Herds } & \multirow{2}{*}{ No. of cows } & \multicolumn{2}{c}{ Brucella isolates } \\
\cline { 3 - 4 } & & Smooth & Rough \\
\hline Herd 1 & 6 & 0 & 2 \\
Herd 2 & 12 & 0 & 3 \\
Herd 3 & 8 & 0 & 0 \\
Total & 26 & 0 & 5 \\
\hline
\end{tabular}

Medium. Tryptic soya agar (Difco Laboratories, Detroit, MI, USA) was used for culturing of brucella organisms. Isolation, identification and typing of Brucella abortus RB51 vaccinal strain: Isolates were typed according to (Alton et al., 1988).

\section{Results \\ Identification of the rough Brucella isolates. \\ 1- Isolates did not contain A or M antigens. \\ 2- Isolates were not lysed by Tibilisi phage. \\ 3- Isolates were resistant to rifampicin. \\ 4- Agglutination with acriflavin and staining with crystal violet indicated rough colonies.}

\section{Discussion}

In this study, a total of 3810 cattle in three dairy herds including heifers, pregnant and nonpregnant cows were vaccinated by the RB51 vaccine for four successive years as these herds were suffering from brucellosis and quarantined under the supervision of the veterinary authorities. Among the vaccinated pregnant cows, 26 cows representing the three herds were aborted 45-67 days post-vaccination in the year 2005, (Table 1).

Serological examination of aborted cows two weeks after abortion revealed no brucella antibodies using TAT, BAPAT and Rivanol test indicating that cows had not been exposed to field brucella infection. On the other hand, using a rough antigen of RB51, positive results were obtained with a mean titer of $1 / 640$ in $6(0.62 \%)$ cows of herd (1) and 12 (1.25\%) cows of herd (2) and $1 / 320$ in $8(0.83 \%)$ cows of herd (3). These titers were related to the activity of Brucella abortus RB51 vaccinal strain.

Attempts to isolate brucella organisms from vaginal discharges and milk samples of aborted cows revealed detection of 5 brucella isolates from the vaginal exudates of 5 aborted cows.

These isolates were typed as Brucella abortus biovar1 RB51 rough strain. This indicates that the vaccinal strain of RB51 reached the uterus and caused placentitis with subsequent abortion and shedding of the vaccinal strain. Brucella abortus RB51 could be isolated from milk and vaginal exudates of parturient cows 60 days post-vaccination, (Arellano-Reynoso et al., 2004). In addition, in Mexico, RB51 vaccinal strain could be isolated 
from milk of parturient cows postvaccination, (Leal et al., 1999). Such findings and those reported by the other authors indicated the possibility of exten-sive bacterial multiplication of RB51 vaccinal strain in vaccinated cattle with shedding of the microorganism resembling the phenomenon corresponding to that occurs in case of Brucella abortus strain S19 vaccination of adult cows. Here, it is worthy to mention that in USA, vaccination of pregnant cows can only be done under authorization and in Mexico it is done with reduced dose of RB51. Concerning the cases of abortion recorded in this study, pregnant cows were vaccinated with the full dose of RB51 vaccine.

Abortion associated with isolation of RB51 vaccinal strain from recently vaccinated cows in this study served as evidence that the vaccinal strain is the cause of abortion. In contrast no brucella isolates could be recovered from vaccinated non-aborted cows. Shedding of the vaccinal strain may result in animals and human infections. Interestingly, human infection due to RB51 cannot be diagnosed by the current serological tests, which detect antibodies against smooth antigen.

Isolation of rough RB51 vaccinal strain from the vaginal exudates of aborted recently vaccinated cows in this study is considered the first record under field conditions; therefore, it is of utmost importance to investigate in future studies whether the shed vaccinal strain could display a virulent phenotype allowing for intracellular multiplication.

\section{References}

Alton, G. G.; Jones, L. M.; Angus, R. D. and Verger, J.M. (1988): Techniques for the brucellosis laboratory. INRA publication, Paris, France.

Arellano-Reynoso; Diaz-Aparicio, E.; Leeal, M.H.; Hernadez, L. and Grovel, J. (2004): Intracellular trafficking study of RB51 B. abortus vaccinal strain isolated from milk. Vet. Microbiol., 98: 307.

Blasco, J. M. (1998): Profilaxis vacunal de la brucellosis. memorias de III foro national de brucellosis. Acapulco, Mexico.

Covert, J.; Eskra, L. and Splitter, G. (2005): Isolation of Brucella abortus total RNA from B. abortus infected murine RAW macrophages. J. Microbiol. Meth., 60: 383.

EL-Diasty M. (2004): Epidemiological and immunological studies on bovine brucellosis. MVSc. Thesis, Infect. Dis., Fac. Vet. Med., Suez Canal Univ., Egypt.

Hosein, H. I.; Montasser, A. M.; EL-Sherif, A. M. and Soliman, H. S.; (2002): Evaluation of the efficacy of the mutant rough Brucella abortus RB51 vaccine in guinea pigs $10^{\text {th }}$ Sci. Cong. Fac. Vet. Med.

Hosien, H. I.; Sohair, Y.; Enany, M. and Gabal, M. A. (2001): The role of some Brucella carriers (stray dogs and cats) in maintenance of Brucella infection. Beni-Suef Vet. Med. J., 2: 521-529.

Leal, H. M.; Diaz, E.; Hernandez, L. and Suraez, F. (1999): Br.abortus RB51 vaccination and revaccination in cattle introduced in a flock with a brucellosis outbreak. Proc. $50^{\text {th }}$ Ann.Meeting Conf. Res. Works Anim. Dis. Chicago. IL, USA.

Mehanna, A. M. A. (1989): Comparative studies on brucella antigens prepared by different methods. MVSc. Microbiol., Fac. Vet. Med., Cairo Univ., Egypt.

Schurig, G. G.; Roop, I. I.; Bagchi, T.; Boyle, S.; Buhrmain, D.; and Sriranganthan, N.; (1991): Biological properties of RB51, Stable rough strain of B. abortus. Vet. Micobiol., 28:171.

Schurig, G. G.; Sriranganthan, N. and Corbel, M. J. (2002): Brucellosis vaccines past, present and future. Vet. Microbiol., 90:479. 